\title{
Proyecto para el reconocimiento, fortalecimiento y fomento de las cocinas tradicionales de la provincia de Gutiérrez, Boyacá
}

\section{Project for the recognition, strengthening and promotion of the traditional kitchens of the Gutierrez province, Boyacá}

\author{
Angie Lorena Hernández Bolaños* \\ Fundación Universitaria San Mateo \\ Bogotá, Colombia
}

\section{Camila Andrea Muñoz*}

Fundación Universitaria San Mateo

Bogotá, Colombia

\section{Robert Fredy Maldonado***}

Fundación Universitaria San Mateo

Bogotá, Colombia

\section{Guillermo Ramírez****}

Fundación Universitaria San Mateo

Bogotá, Colombia

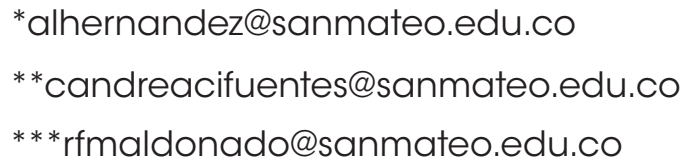

Articulo de investigación

Recepción: 30 de julio de 2019. Aceptación: 21 de agosto de 2019

Cómo citar este artículo

Hernández, A; et al. (2019). Proyecto para el reconocimiento, fortalecimiento y fomento de las cocinas tradicionales de la provincia de Gutiérrez, Boyacá. Sosquua 1(1), 97-106. Recuperado a partir de http://cipres.sanmateo.edu.co/index.php/sosquua 


\title{
Resumen
}

El presente artículo se enfoca en la investigación, salvaguardia y fortalecimiento de la alimentación y la cocina tradicional con comunidades de la provincia Gutiérrez en el departamento de Boyacá, Colombia. Allí, se buscará sensibilizar a la población sobre el problema que representa el hecho de perder sus tradiciones culinarias para los miembros del territorio.

Pallabras clave: cocina tradicional, salvaguardia, soberanía alimentaria, rescate culinario.

\begin{abstract}
The present paper is focused on inquiry, safeguarding and strengthening of food and traditional cuisine with communities of the Gutiérrez, a province in Boyacá, Colombia. This will seek to sensitize the population about the problem that represents the fact of losing their culinary traditions for the members of that territory.
\end{abstract}

Keywords: traditional cuisine, safeguard, food sovereignty, culinary rescue. 


\section{Introducción}

La cocina tradicional hace parte fundamental de la descripción de un pueblo, ciudad, región o país. Dichos elementos deben ser analizados en la generación y el reconocimiento de una identidad, donde el arraigo de sus pobladores debe estar establecido bajo las normas y tradiciones del lugar de análisis.

Para ello se inicia el proceso de investigación en la provincia de Gutiérrez, en el departamento de Boyacá, que busca el fomento y fortalecimiento de las cocinas tradicionales. Allí se aborda el aporte del área de la gastronomía como desarrollo de reconocimiento del valor ancestral dentro del territorio, como medio de difusión a nivel nacional e internacional de la cultura de la región (Nyéléni, 2007).

A lo largo del documento se puede evidenciar el trabajo de investigación y de campo realizado en la provincia, con el fin de salvaguardar el patrimonio cultural intangible gastronómico de la población. Bajo la ley nacional, esta busca "entender la cocina tradicional en un país multicultural, pluriétnico y biodiverso como el nuestro, es fundamental adentrarse en las cocinas para conocer la esencia de sus poblaciones" (Ministerio de Cultura, 2012).

A partir de los establecido por el Ministerio de Cultura, y mediante el desarrollo del trabajo en campo, se puede evidenciar que la población no ha proyectado sus costumbres debido a las nuevas técnicas de cocina que, como consecuencia, dejan de lado las preparaciones autóctonas. Lo anterior genera cambios alimentarios y ha conllevado a una transformación en la relación de la comunidad con la naturaleza, la cultura y la tecnología (Chavarrías, 2008). 


\section{Resultados}

Las cocinas tradicionales son un componente importante dentro de la cultura de los municipios que componen la provincia Gutiérrez, en Boyacá. En ella se cohesionan la identidad de sus pobladores con las tradiciones, que han sido transmitidas por generaciones, y los saberes culinarios, que involucran prácticas agrícolas y sociales que durante muchos años ayudaron a formar la identidad del territorio (Alcaldía de El Cocuy, 2014). Desafortunadamente estas costumbres han cambiado y ahora están en riesgo. Lo anterior ha causado, en algunos casos, un agudo desconocimiento de su propio patrimonio culinario, originando el cambio en las prácticas tradicionales de transformación, conservación, manejo y consumo de alimentos Ministerio de Cultura, 2004).

Esta investigación quiere salvaguardar y fortalecer la cocina tradicional de este territorio. Los cambios en las costumbres alimenticias de sus pobladores han causado una desvalorización de la propia cultura alimentaria. En la actualidad, la cocina tradicional es tratada por las nuevas generaciones como cocina rudimentaria, poco elaborada o como comida de pobres o de campesinos, para abrir paso a nuevas costumbres alimentarias que son industrializadas. En consecuencia, ha generado profundos cambios en la vida social. Aún así, algunos casos han significado cambios positivos para la comunidad, pero en otros ha causado un detrimento en el patrimonio cultural inmaterial del territorio.

El patrimonio cultural inmaterial está constituido, entre otros, por las manifestaciones, usos, practicas, representaciones, expresiones, conocimientos, técnicas y espacios culturales, que las comunidades y los grupos reconocen como parte integrante de su patrimonio cultural (Ley 397, 1997, art. 11.1). 


\section{Preparaciones gastronómicas}

El maíz tostado y la harina de maíz le disputaban al cazabe el primer lugar en los suministros preferidos por los conquistadores, a la hora de emprender largos viajes de conquista. En las exploraciones, los soldados llevaban tanta harina de maíz como se pudiere pues era ingrediente esencial para hacer sus mazamorras, que era su sustento (Buitrago, s.f.).

En sus diferentes formas y preparaciones, el maíz y el trigo son las bases de la alimentación de las poblaciones de la provincia. En esta lista de preparaciones se puede notar una gran variedad de sopas, arepas y amasijos.

\section{Boyacá:}

\section{Sopas:}

- De zarazo

- Sopa de maíz pintado

- Sopas de agrio

- Cuchuco de trigo, cebada y maíz

- Ruyas de maíz tostado

- Ruyas de maíz desmoronado

- Ruyas de maíz desmoronado

Figura 1. Estufa de leña.

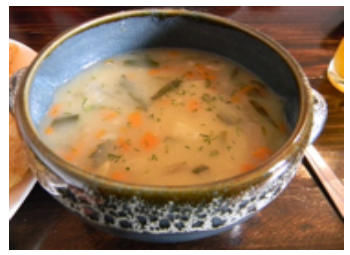

Fuente: Guillermo Ramírez, Guacamayas, Boyacá, 2017.
- Ruyas escucuridas de maíz

- Ruyas de trigo

- Sopa de pan

- Sopa de chorotas

- Angú

- Sopa de leche

- Los mutes de maíz y frijol

Figura 2. Ajiaco montañero o boyacense.

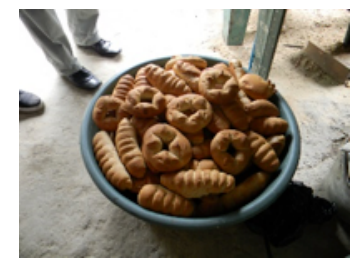

Fuente: Guillermo Ramírez, Güicán de la Sierra, Boyacá, 2017. 


\section{Platos fuertes:}

- Cocido boyacense

- Chivo sudado

- Carne asada

- Gallina asada con machucado de papa

Figura 3. Tamal.

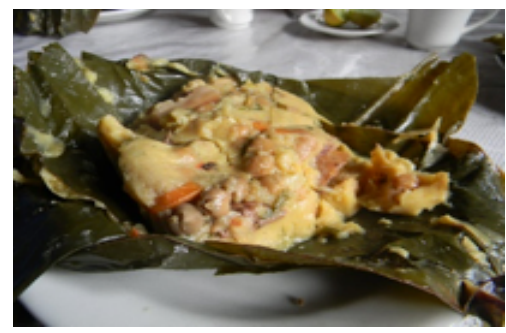

Fuente: Guillermo Ramírez, Cocuy, Boyacá, 2017.

\section{Amasijos:}

- Arepa de maíz dulce

- Arepa de maíz con asadura de cerdo

- Arepa zarapa rellena de queso

- Arepa de maíz de morona

- Arepa tiesa: pan ázimo

- Arepa liuda

- Arepa frita

- Arepa corrida

- Almojábanas
- Chivo asado

- Tamales

- Hallacas boyacenses

Figura 4. Molino manual.

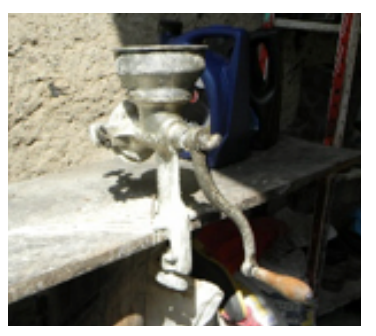

Fuente: Guillermo Ramírez, Guacamayas, Boyacá, 2017.

- Empanadas boyacenses

- Pastel de papa

- Machucado

- Mantecadas de huevo criollo

- Moronitas de maíz

- Envueltos de maíz

- Moronitas de maíz

- Envueltos de maíz

- Cucas 
\begin{tabular}{l|l} 
- Colaciones & - Mestizas de chicharrón \\
- Plumeros &
\end{tabular}

Figura 4. Molino manual.

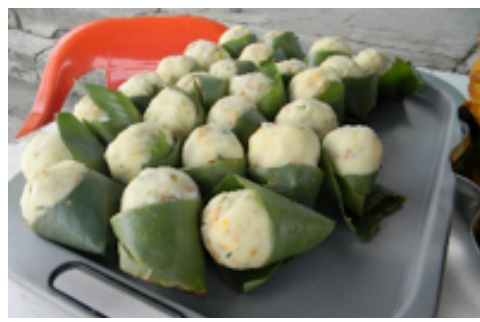

Fuente: Guillermo Ramírez, Guacamayas, Boyacá, 2017.

Figura 4. Molino manual.

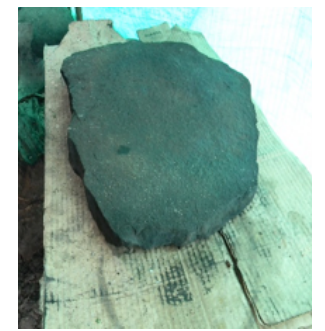

Fuente: Guillermo Ramírez, Guacamayas, Boyacá, 2017.

\section{Bebidas:}

- Chicha

- Masato

Figura 4. Masato de bollo con queso doble crema.

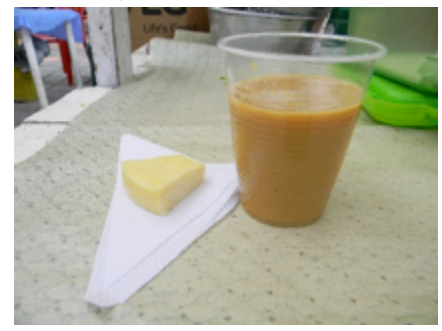

Fuente: Guillermo Ramírez, Boavita, Boyacá, 2017.
Figura 4. Molino manual.

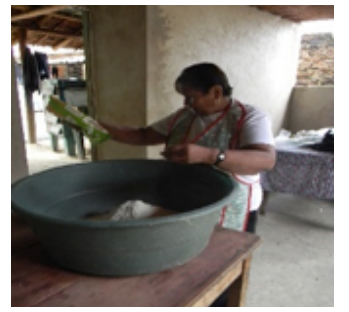

Fuente: Guillermo Ramírez, Guacamayas, Boyacá, 2017.

Figura 4. Molino manual.

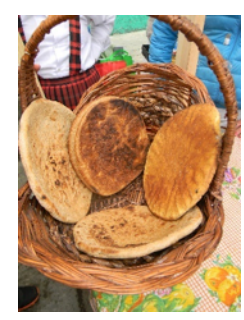

Fuente: Guillermo Ramírez, Guacamayas, Boyacá, 2017.

- Caspiroleta

- Masato de envuelto

Figura 4. Canasta de Panes.

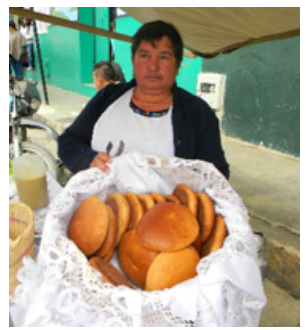

Fuente: Guillermo Ramírez, Güicán de la Sierra, Boyacá, 2017. 


\section{Discusión de resultados}

Para llevar a cabo esta investigación para el conocimiento, fortalecimiento y fomento de las cocinas tradicionales de la provincia de Gutiérrez, en Boyacá, se llevan a cabo diversas actividades. Se inicia con una investigación de datos generales de cada uno de los municipios que conforman la provincia. Esta investigación se recopila mediante elementos de gentilicios, economía, patrimonios, entre otros, que se quiera conocer por medio de su página oficial.

A partir de esta información se concluye que la mayoría de la actividad económica recae en la agricultura. De ahí que se decida investigar el comportamiento que han tenido los cultivos plantados por los habitantes, a lo largo de los años (Acosta \& Amylkar, 2014). En esta medida, se consulta la página de agro net donde, por producto, muestra datos como área de cosecha, producción por toneladas y participación área cosecha nacional. En este punto ya se da cuenta de uno de los problemas y cambios que ha enfrentado esta provincia: su producción ha disminuido considerablemente.

Si bien es cierto que Boyacá es uno de los principales departamentos que se sustenta a sí mismo y al resto del país a través de la agricultura, ¿por qué disminuyó el aporte de la provincia al país? Tanto sus habitantes como los medios de comunicación cuentan que esta provincia fue víctima del terrorismo por las Fuerzas Armadas Revolucionarias de Colombia (FARC), el Ejercito de Liberación Nacional (ELN) y el paramilitarismo. Allí hubo secuestros, masacres y desplazamiento forzoso. Además de esto obligaron a los campesinos, y a sus familias, a convertir los cultivos ilícitos en su sustento económico. Entonces ya no se cultivaban alimentos sino coca. Luego de que fueran libres, no todo fue igual y sus cultivos no volvieron a ser los mismos. En la actualidad la mayoría solo cultiva para su sustento diario y ya no venden, como sucedia anteriormente. 


\section{Conclusiones}

A lo largo de la investigación se evidencia la falta de información de la cultura de la región de la provincia de Gutiérrez, en cualquier medio, ya sea físico o digital. Al revisar minuciosamente las estadísticas de los cultivos en Boyacá, se infiere que la producción agricola ha bajado significativamente, especialmente en la provincia de Gutiérrez. El conflicto armado que sufrió el territorio influyó drásticamente en la producción agrícola debido a que los terrenos de cultivo eran destinados para actividades ilícitas como la siembra de coca.

\section{Lista de referencias}

Acosta, M. \& Amylkar, D. (2014). seguridad alimentaria. Recuperado de https://www.elheraldo.co/columnas-de-opinion/la-seguridad-alimentaria-170910

Alcaldía de El Cocuy. (2014). Sitio oficial de El Cocuy en Boyacá, CoIombia. Recuperado de http://www.elcocuy-boyaca.gov.co/presentacion.shtml

Buitrago, V. (s.f). Gastronomía y arte culinario. Recuperado de http:// www.boyacacultural.com/index.php?option=com_content $\&$ view $=$ article $\& i d=44 \& \mid$ temid $=45$

Chavarrías, M. (2008). Globalización y seguridad alimentaria. Recuperado de: http://www.consumer.es/seguridad-alimentaria/sociedad-y consumo/2008/03/24/175595.php

Congreso de Colombia. (7 de agosto de 1997) Ley general de cultura. [Ley 397 de 1997].

Ministerio de Cultura. (2004). Estrategia para el fortalecimiento de las capacidades de apropiación de la política de salvaguardia del $\mathrm{PCl}$. Bogotá: Ministerio de Cultura. 
Nyéléni, S. (2007). Soberanía alimentaria. Recuperado de http://www. soberaniaalimentaria.tv/

Ministerio de Cultura. (2012). Política para el conocimiento, la salvaguardia y el fomento de la alimentación y las cocinas tradicional. Bogotá: Ministerio de Cultura. 